\title{
Tratamento térmico do silano para melhorar a cimentação adesiva de restaurações cerâmicas odontológicas
}

\section{(Silane heat treatment to improve cementation of ceramic dental restorations)}

\author{
L. M. Peixoto F. , E. Batitucci', C. B. S. Daroz ${ }^{2}$, H. R. Sampaio F. \\ ${ }^{I}$ Faculdade de Odontologia da Universidade do Estado do Rio de Janeiro - UERJ, Boulevard 28 de Setembro \\ 157, $2^{\circ}$ andar, sala 10, Pavilhão Mario Franco Barrozo, Vila Isabel, Rio de Janeiro, RJ 20551-030 \\ ${ }^{2}$ Faculdade de Odontologia da Universidade Federal do Espirito Santo - UFES, Av. Marechal Campos 1468, \\ Hospital das Clínicas, Departamento de Prótese, UFES, Vitória, ES 29040-091 \\ leticia.m.peixoto@hotmail.com,edubatitucci@uol.com.br, \\ claudiabatitucci@yahoo.com.br,hsampaiofilho@gmail.com
}

\begin{abstract}
Resumo
O sucesso da cimentação adesiva para restaurações cerâmicas odontológicas está, em parte, relacionado com a correta indicação de um tratamento superficial do substrato cerâmico. Diversos tipos de tratamento da superfície interna das restaurações cerâmicas têm sido propostos na literatura, dentre os quais se destacam o condicionamento com ácido hidrofluorídrico e a silanização. Todavia, o condicionamento com ácido pode reduzir a resistência da cerâmica dependendo da extensão de remoção da fase cristalina, além de exigir grande cautela durante manuseio deste tipo de ácido por ser um material altamente tóxico. Com a possibilidade de otimizar a efetividade da silanização pelo seu aquecimento no momento da evaporação, surge em vista uma nova possibilidade na Odontologia; a de suprimir a utilização do ácido hidrofluorídrico como condicionador para tratamento de superfície das cerâmicas no consultório odontológico e junto com ele os seus riscos a saúde humana em virtude de sua alta toxicidade. Desta forma, o objetivo desta revisão de literatura é abordar o tratamento térmico do silano como forma de melhorar a cimentação adesiva de restaurações cerâmicas.

Palavras-chave: cerâmica, resistência adesiva, silano.
\end{abstract}

\begin{abstract}
The success of the restorative dental ceramic cementation is partly related to the correct indication of a surface treatment of the ceramic substrate. Several types of treatment have been proposed in the literature, among which is hydrofluoric acid etching and silanization. However, acid etching can reduce the strength of the ceramic depending on the extent of crystalline phase removal, and this type of acid require great care in handling because its high toxicity. With the possibility to optimize the effectiveness of silane by heating them at the time of evaporation, there is a new possibility in mind in dentistry, to suppress the use of hydrofluoric acid as a conditioner for ceramic surface treatment in the dental office and with it the their risks to human health because of its high toxicity. Thus, the purpose of this review is to address the thermal treatment of the silane as a way to improve the adhesive cementation of ceramic restorations.
\end{abstract}

Keywords: ceramics, microtensile strength, silane.

\section{INTRODUÇÃO}

A cimentação adesiva para restaurações cerâmicas odontológicas pressupõe a obtenção de um corpo único final formado pela união entre a restauração e a estrutura dentária remanescente, o que permite uma melhor distribuição das cargas durante a mastigação, diminuindo o risco de fratura da restauração [1]. O sucesso desta união está, em parte, relacionado com a correta indicação de um tratamento superficial do substrato cerâmico [2-5]. Diversos tipos de tratamento da superfície interna das restaurações cerâmicas têm sido propostos na literatura, sempre de acordo com a composição química e microestrutura do material cerâmico, visando produzir retenções micromecânicas que auxiliem no processo de adesão. $\mathrm{O}$ condicionamento com ácidos tem sido proposto, sendo que o ácido hidrofluorídrico tem se mostrado efetivo sobre cerâmicas ricas em sílica [5-7]. Sua aplicação aumenta a rugosidade de superfície do material pela remoção da fase cristalina, melhorando a retenção micromecânica e produzindo características morfológicas do tipo favo de mel sobre a superfície da cerâmica [8]. Todavia, sua utilização pode reduzir a resistência da cerâmica dependendo da extensão de remoção da fase cristalina, além de exigir grande cautela durante manuseio deste tipo de ácido por ser uma material altamente tóxico $[9,10]$. A silanização é um outro importante tratamento superficial que vem sendo estudado. O silano é o agente que garante a adesão química dos componentes inorgânicos da cerâmica à porção orgânica do cimento resinoso. Ele é um monômero no qual o silício está ligado a radicais orgânicos reativos e a grupamentos 
monovalentes hidrolisáveis. Os radicais orgânicos reativos ligam-se quimicamente com as moléculas de resina, como Bis-GMA e TEGMA, encontrados tanto no adesivo como no cimento resinoso [11]. Por essas características, tem sido proposta sua utilização para otimizar os resultados clínicos na cimentação de restaurações indiretas de resina e de cerâmica, assim como nos reparos desses trabalhos na cavidade oral. Ainda em relação aos silanos, o tratamento térmico deste monômero permite a remoção de películas externas, deixando apenas a camada mais interna que é mais estável e quimicamente unida à cerâmica. Desta forma, a utilização do silano termicamente tratado permite a melhora da união entre resinas compostas e a sílica das cerâmicas vítreas [12].

Com a possibilidade de otimizar a efetividade da silanização pelo seu aquecimento no momento da evaporação, surge em vista uma nova possibilidade na Odontologia; a de suprimir a utilização do ácido hidrofluorídrico como condicionador para tratamento de superfície das cerâmicas no consultório odontológico e junto com ele os seus riscos a saúde humana em virtude de sua alta toxicidade. Desta forma, o objetivo desta revisão de literatura é abordar o tratamento térmico do silano como forma de melhorar a cimentação adesiva de restaurações cerâmicas. A base de dados www. periodicos.capes.gov.br foi utilizada buscando artigos publicados de 1969 a 2012 com os seguintes unitermos: airdrying, evaporation, silane, ceramic, surfacetreatment.

\section{REVISÃO DE LITERATURA}

$\mathrm{Na}$ Odontologia os silanos podem ser usados como prétratamento de superfícies, funcionando como agentes de acoplamento para aderir à cerâmica a um compósito em restaurações dentárias; em reparos intra-oral de superfícies cerâmicas ou de resinas e para acoplar uma camada bioinerte sobre implantes de titânio [13]. Essa ampla aplicação justifica-se na sua capacidade de funcionar como mediadores que promovem a adesão entre matrizes orgânicas e inorgânicas através de sua dupla reatividade. A dupla reatividade permite que o grupo funcional não hidrolisável com um carbono de dupla ligação possa polimerizar com monômeros de resina composta contendo duplas ligações; e o hidrolisável, grupo alcoxi (por exemplo, metoxi-O-CH3, etoxi-O-CH2CH3) possa reagir com o grupo hidroxila rico na superfície da cerâmica [13].

A aplicação do silano sobre a superfície da cerâmica, previamente tratada ou não, tem o objetivo de melhorar a união entre esta e o cimento resinoso. Sua atuação seria tanto física, por aumentar o molhamento da superfície da cerâmica tornando-a mais receptiva ao adesivo, quanto química, unindo-a ao cimento à semelhança da união entre a partícula inorgânica e a matriz orgânica quando da fabricação das resinas compostas [14]. Várias pesquisas ao longo dos anos têm sido realizadas com intuito de avaliar o papel exercido pelos silanos na resistência de união, assim como na durabilidade da união, dos cimentos resinosos às superfícies cerâmicas. Em 1969, foi analisada a resistência de união da porcelana tratada com silano e unida a resinas acrílicas de polimerização química e térmica, tendo concluído que o silano melhora significativamente a resistência de união desses materiais [15]. Já em 1978, foi verificada a resistência de união da porcelana tratada com silano e unida à resina composta, concluindo que o tratamento da porcelana com silano produz união eficaz, sendo também um método efetivo para reparo de restaurações de porcelana com resina composta na cavidade oral [16]. Em 1984, foi observado o efeito de agentes de silanização na resistência de união à tração da superfície da cerâmica condicionada [17]. Observou-se que os melhores resultados foram obtidos combinando o condicionamento ácido com o agente de silanização, uma vez que o condicionamento da superfície cerâmica cria micro-porosidades que promove a retenção mecânica do adesivo, e a aplicação de um agente de união silano aumenta ainda mais essa resistência de união. O condicionamento efetivo da superfície interna era, então, considerado essencial para o sucesso das restaurações cerâmicas. Já em 1992, foi investigado o efeito de vários tratamentos de superfície na resistência de união entre resina composta e a cerâmica aluminizada Vitadur N (Vita) [18]. Concluiu-se que o agente de silanização tem um importante papel na união entre a resina composta e a cerâmica; e que a combinação do ácido hidrofluorídrico a $2 \%$ e o agente de silanização potencializa essa união. Estes achados foram confirmados em estudos posteriores [7, 19-21]. Apesar da combinação de ambos os procedimentos ter alcançado os melhores resultados, salientou-se para os cuidados que devem ser tomados durante o uso clínico do ácido fluorídrico, referentes ao tempo de aplicação e manipulação do produto. Um estudo avaliando reparo intra-oral de restaurações cerâmicas questionou se o ácido fluorídrico (AF), em função da periculosidade de sua utilização intra-oral, não poderia ser substituído pelo flúor fosfato acidulado (FFA) [22]. O efeito observado após a aplicação do FFA pareceu não ser suficiente para promover retenção micromecânica efetiva da resina à superfície cerâmica em comparação com as amostras condicionadas com AF; mostrando que quanto maior tempo de aplicação mais evidente e agressivo era o padrão de condicionamento da superfície cerâmica. Apesar do condicionamento ácido aumentar a rugosidade superficial, e assim melhorar a retenção mecânica, o tempo de condicionamento e concentração do ácido são fatores que deverão ser bem controlados, pois, com o aumento da concentração ou do tempo de ação do ácido sobre a superfície, pode promover a dissolução parcial dos cristais dentro da matriz vítrea da cerâmica. Dessa forma o AF, apesar de criar uma superfície retentiva, enfraquece de forma significativa a superfície cerâmica [23, 24]. A utilização do AF como condicionador para as cerâmicas em consultório odontológico pode ser um fator de risco a saúde humana em virtude de sua alta toxicidade. Este composto químico em contato com tecido humano pode provocar dor imediata e queimadura superficial, com eritema, vesiculação e necrose. A concentração utilizada em consultório odontológico geralmente pode levar algumas horas até o estabelecimento de 
sintomas. Contudo, trabalhos da área médica consideram que regiões atingidas superiores a $2 \%$ do total do corpo humano como extremamente grave, podendo ser fatais. Contato com os olhos apresentam severidade pela lesão imediata de córnea. Além disso, a inalação pode ocasionar edema agudo de pulmão dependente da quantidade e concentração do produto [25, 26]. Em função desses fatores negativos, vários estudos que avaliam diferentes formas de tratamento térmico do silano vem sendo realizados com intuito de aumentar a resistência da união entre a resina composta e a cerâmica e assim possivelmente suprimir o condicionamento com AF. Para acelerar o mecanismo de interação química entre o silano e superfícies inorgânicas, a reação pode ser catalisada por tratamento ácido ou aquecimento. O tratamento térmico de vidros silanizados é realizado rotineiramente na indústria de vidros para maximizar a resistência de união. A secagem com ar quente aumenta a eficácia de alguns silanos quanto utilizados como agentes de união entre cerâmica e compostos resinosos. A alta temperatura do silano (70-80 ${ }^{\circ} \mathrm{C}$ ) não é viável para procedimentos em consultório, mas uma corrente de ar quente de aproximadamente $38^{\circ} \mathrm{C}$ pode ser utilizada para este fim [27].

Vários estudos [12, 14, 28-30] tem sido realizados ao longo dos anos acerca do tratamento térmico do silano, tendo sido demonstrado que estes tratamentos realizados de formas diversas podem interferir na orientação do filme de silano, na constituição, estabilidade e resistência de união a determinados substratos, uma vez que permite a remoção das películas externas, deixando a camada mais estável e quimicamente reativa. Em 1994, um estudo descreveu a orientação de filmes de poli (dimethy1 silano) preparados pela evaporação [28]. As amostras foram preparadas em diferentes velocidades de evaporação e temperaturas de substrato. Após a evaporação por aquecimento o polímero foi recristalizado sobre o substrato. A estrutura e orientação dos filmes foram investigadas utilizando difração de raios $\mathrm{X}$ de grande-angular e método de absorção ultravioleta. Quando analisada a velocidade de evaporação, verificou-se que os filmes preparados em velocidade de evaporação alta (maior do que $190 \mathrm{~nm} \cdot \mathrm{min}^{-1}$ ) tem a maioria das cadeias de silício paralela à superfície do substrato; que os filmes preparados em velocidade de evaporação intermediária (de 28-40 nm. $\mathrm{min}^{-1}$ ) tem algumas das cadeias de silício inclinadas na direção da superfície; e que o filmes preparados em velocidade de evaporação baixa (inferior a $20 \mathrm{~nm} \cdot \mathrm{min}^{-1}$ ), as cadeias de silício se encontram perpendiculares à superfície do substrato. Essa diferença de orientação do filme pode ser explicada pelo fato de que com velocidade de evaporação lenta, no processo de recristalização, as cadeias de silício vaporizadas se movem para posições mais estáveis, sendo que com velocidade de evaporação elevada, as cadeias de silício não conseguem mover-se a uma distância suficiente. Na etapa do estudo em que foi analisado a evaporação do filme em diversas temperaturas de substrato (com velocidade de evaporação constante em $33 \mathrm{~nm} \cdot \mathrm{min}^{-1}$ ), pôde-se observar que o filme preparado em temperatura de substrato baixa $\left(13^{\circ} \mathrm{C}\right)$ não mostrou uma orientação particular, enquanto que o filme preparado em temperatura de substrato alta as cadeias de silício apresentam-se perpendiculares a superfície do substrato. Isso justifica-se pela alta temperatura do substrato em permitir a movimentação das cadeias de silício por uma distância suficiente para criar cristais bidimensionais. Desta forma, com este estudo pode ser afirmado que cadeias de sílica do poli (dimetil silano) são orientadas perpendicularmente a superfície do substrato quando o filme é preparado com baixa velocidade de evaporação e alta temperatura do substrato.

Em 1995 foram investigados os efeitos das condições de tratamento e estocagem na resistência de união ao cisalhamento entre cerâmica/compósito. Observouse que superfícies das cerâmicas Dicor ${ }^{\circledR}$ (Dentsply), Mirage $^{\circledR}$ (Mirage) e Vitabloc $^{\circledR}$ (Vita) que receberam os seguintes tratamentos de superfície: a) lixa de granulação $600 ;$ b) jateamento com óxido de alumínio $50 \mu \mathrm{m}$ por $3 \mathrm{~s}$; c) condicionamento ácido com gel de amônia ou ácido hidrofluorídrico por $90 \mathrm{~s}$ e a aplicação de um dos três distintos silanos: 1) 2,5\% em volume de $\gamma$-metacriloxipropiltrimetoxisilano, $2,5 \%$ em volume de ácido acético misturado com $95 \%$ de etanol; 2) $2,5 \%$ em volume de $\gamma$-metacriloxipropiltrimetoxisilano, $0,5 \% \mathrm{em}$ volume de $\mathrm{N}, \mathrm{N}$ dimetilparatoluedina e $2,5 \%$ em volume de ácido acético misturado com 94,5\% em volume de etanol; 3) $2,5 \%$ em volume de viniltriclorosilano e $2,5 \%$ em volume de ácido acético misturado com $95 \%$ de etanol que foram secos: a) à temperatura ambiente por $60 \mathrm{~s} \mathrm{ou} \mathrm{b)}$ à $100^{\circ} \mathrm{C} \pm 5{ }^{\circ} \mathrm{C}$ com jato de ar quente por $60 \mathrm{~s}$ e resfriados à temperatura ambiente. Concluiu-se que a retenção mecânica parece ser o fator principal que influencia a resistência de união da cerâmica/compósito. $\mathrm{O}$ tratamento térmico a $100{ }^{\circ} \mathrm{C}$ por $60 \mathrm{~s}$ na superfície tratada com silano (metacriloxipropiltrimetoxisilano) dobrou a resistência de união ao cisalhamento, o que poderia ser atribuído à eliminação de água e outros contaminantes, possibilitando a formação de uma ligação covalente silano-sílica. Essa evaporação também aumentou o número de sítios de união disponíveis para reagir com o silano [14]. Em 2002, foi realizado um estudo com o objetivo de explorar os métodos para melhorar a resistência de união da cerâmica-silanoresina, de forma que fosse possível eliminar o processo de condicionamento ácido dos materiais cerâmicos com ácido fluorídrico. Para isto foram testadas diferentes formas de aplicação e tratamentos térmicos do silano com intuito de eliminar subprodutos pela remoção das camadas mais externas do silano (1-imersão no silano por $60 \mathrm{~s}$ e secagem com jato de ar à temperatura ambiente (15 s); 2- imersão por $60 \mathrm{~s}$ e secagem em forno a $100{ }^{\circ} \mathrm{C}(2 \mathrm{~min})$; 3- aplicação do silano com microbrush (60 s) e secagem com jato de ar à temperatura ambiente (15 s); 4- aplicação com microbrush (60 s) e secagem a $100{ }^{\circ} \mathrm{C}$ (2 min); 5-aplicação com microbrush $(60 \mathrm{~s})$ e secagem com ar quente a $50 \pm 5^{\circ} \mathrm{C}(15$ s); 6- semelhante ao 5 , seguido de enxágüe com água em ebulição (15 s) e secagem com ar quente (30 s); 7-semelhante ao 6 , aplicando-se uma fina camada de resina sem carga antes da cimentação com cimento resinoso). Neste estudo foram 
realizados testes de ciclagem térmica, resistência a tração, e análise do padrão de fratura. Observou-se que a aplicação do silano com pincel em vez da imersão do espécime promoveu uma camada mais fina; que o tratamento com calor eliminou água, álcool e outros subprodutos auxiliando na reação de condensação silano-sílica, promovendo a formação de uma ligação covalente; que a secagem com ar quente a $50{ }^{\circ} \mathrm{C}$ foi preferível sobre a secagem com $100{ }^{\circ} \mathrm{C}$; e que o enxague com água em ebulição aumentou muito o número de fraturas coesivas no cimento, provavelmente pela remoção da camada mais externa da película de silano. Concluiu-se que uma adesão durável e adequada entre a cerâmica feldspática reforçada por leucita e o cimento resinoso poderia ser obtida pela otimização dos procedimentos de silanização, sem necessidade de retenções micromecânicas [12]. Esse estudo explica que devido à natureza química do agente de união silano com a presença de solventes à base de álcool, a forma como a evaporação ocorre após sua aplicação na superfície da cerâmica poderia gerar diferentes resultados da resistência de união. Após a secagem, não se observa uma monocamada de silano, mas uma interface com três diferentes estruturas: uma camada mais externa de oligômeros pequenos que são adsorvidos ao vidro, passível de ser removida por solventes orgânicos ou por água à temperatura ambiente. Uma segunda região consistiria de oligômeros similares ligados por pontes siloxanas hidrolisáveis por água quente. E na região mais próxima da superfície do vidro as ligações cruzadas são mais freqüentes e uniformes, formando uma rede tridimensional regular, hidroliticamente mais estável. Apenas esta última camada seria necessária para melhorar a adesão. A remoção da camada mais externa da película de silano poderia favorecer a adesão deixando-se apenas a camada mais estável adsorvida quimicamente à superfície da cerâmica.

Um estudo foi realizado com intuito de investigar se a secagem pós silanização com corrente de ar quente poderia aumentar a resistência de união entre compósito resinoso e cerâmico. Com este objetivo confeccionou-se trinta e dois blocos de cerâmica (6 mm x $6 \mathrm{~mm} \times 9 \mathrm{~mm})$, que receberam dois distintos protocolos de silanização por cerâmica. As superfícies foram silanizadas e secas com ar a temperatura ambiente por $1 \mathrm{~min}$ ou ar quente $45 \pm 5^{\circ} \mathrm{C}$ por $2 \mathrm{~min}$. Concluiu-se que a secagem do silano por uma corrente de ar quente foi eficaz para melhorar a resistência de união à tração do compósito a cerâmica [29]. Depois, em 2010 um estudo avaliou a influência dos procedimentos de evaporação de silano na resistência de união a microtração entre uma cerâmica odontológica IPS Empress 2 (Ivoclar Vivadent) e um cimento resinoso quimicamente ativado. Foram confeccionados seis grupos, cada um com três blocos divididos de acordo com o tratamento da superfície cerâmica: dois grupos de controle: NT-sem tratamento, HFS- ácido fluorídrico 10\% mais silano Monobond-S secas à temperatura ambiente $(3 \mathrm{~min})$; os outros quatro grupos foram submetidos a diferentes padrões de evaporação: SRT- silano lavado ( $5 \mathrm{~s})$ e seco a temperatura ambiente (3 $\mathrm{min})$; SBRT- silano lavado em água fervente $(5 \mathrm{~s})$ e seco a temperatura ambiente ( $3 \mathrm{~min}$ ); $\mathrm{SBH}$ silano lavado em água fervente $(5 \mathrm{~s})$ e seco em jato de ar quente a $50 \pm 5{ }^{\circ} \mathrm{C}$ (3 min); SHBRT silano seco em jato de ar quente a $50 \pm 5^{\circ} \mathrm{C}$ (3 min), lavado em água fervente ( $5 \mathrm{~s}$ ) e seco à temperatura ambiente $(3 \mathrm{~min})$. Os padrões de fratura foram analisados em microscópios óptico e eletrônico de varredura. Todos os blocos de NT descolaram durante o corte. O teste One Way ANOVA mostrou maior resistência adesiva para HFS do que para outros grupos. SBRT e SBH foram estatisticamente semelhantes, com maior resistência adesiva do que SRT e SHBRT. As falhas foram $100 \%$ adesivas em SRT e SHBRT. Falhas coesivas dentro da "zona adesiva" foram detectadas em HFS (30\%), SBRT (24\%) e SBH $(40 \%)$. Concluiu-se que o tratamento do silano em todas as condições avaliadas aumenta a resistência de união, mostrando melhores resultados com o condicionamento com ácido HF [30].

\section{DISCUSSÃO}

Diversos tipos de tratamento da superfície interna das restaurações cerâmicas foram propostos na literatura, visando auxiliar o processo de adesão. Vários pesquisadores advogam que as maiores resistências de união foram encontradas usando uma combinação de condicionamento de superfície com ácido hidrofluorídrico e o uso de agentes de união silano $[8,11,31,32]$ observaram que a utilização dos agentes de união silano associados a condicionamento com ácido fluorídrico na concentração de $9,5 \%$ a $10 \%$ por 20 s como tratamento superficial de cerâmicas a base de dissilicato de lítio viabiliza uma boa resistência de união. $\mathrm{O}$ envelhecimento artificial por ciclagem térmica não diminui a resistência de união entre a superfície de uma cerâmica de dissilicato de lítio (IPS Empress $2^{\circledR}$, Ivoclar Vivadent) quando essa associação é realizada [33]. O condicionamento com ácido hidrofluorídrico na superfície de dissilicato de lítio é de grande importância para aumentar a rugosidade superficial dessas cerâmicas, e assim melhorar a retenção mecânica, todavia, o tempo de condicionamento e concentração do ácido são fatores que deverão ser bem controlados, pois, com o aumento da concentração ou do tempo de ação do ácido sobre a superfície, pode ocorrer dissolução parcial dos cristais dentro da matriz vítrea da cerâmica [11]. O tempo de condicionamento ácido da cerâmica a base de di-silicato de lítio recomendado pelo fabricante são de $20 \mathrm{~s}$, o qual foi atestado por diversos estudos [8, 11, 31-33]. Além do ácido hidrofluorídrico ter potencial de enfraquecimento da superficie cerâmica [11, $23,24]$ a sua utilização em consultório odontológico pode causar risco a saúde devido a sua alta [9, 10]. Adicionando esses fatores negativos ao fato de que a resistência de união entre a resina composta e a cerâmica pode ser obtida sem a necessidade da aplicação do condicionamento com ácido hidrofluorídrico sobre a superfície cerâmica, acredita-se que o condicionamento com ácido hidrofluorídrico possa ser suprimido [12]. Para acelerar o mecanismo de interação química entre o silano e superfícies inorgânicas, a reação pode ser catalisada por tratamento térmico (aquecimento). 
A secagem com ar quente aumenta a eficácia de alguns silanos quando utilizados como agentes de união entre cerâmica e compostos resinosos [27]. Vários estudos acerca desses tipos de tratamento tem demonstrado que estes realizados de formas diversas podem interferir na orientação da película de silano, uma vez que permite a remoção das películas externas, deixando a camada mais estável e quimicamente reativa [12], além de reorganizar a camada de silano, uma vez que as cadeias de silicio vaporizadas se movem para posições mais estáveis [28]. Além disso, o tratamento térmico do silano sobre superfícies cerâmicas também viabiliza um aumento da força de união entre a cerâmica e o cimento resinoso [12, 14, 29, 30]. Embora o tratamento térmico da camada de silano venha apresentar bons resultados, ainda sim, estudos mostram a necessidade do condicionamento ácido da superfície cerâmica à base de dissilicato de lítio para obtenção de uma boa resistência de união aos cimentos resinosos.

\section{CONCLUSÕES}

O tratamento térmico do silano previamente a cimentação resinosa de restaurações cerâmicas a base de dissilicato de lítio tem demonstrado bons resultados por possibilitar uma melhor organização e distribuição da película de silano, tornando-a mais estável e quimicamente reativa com material resinoso; porém, de acordo com a literatura, a completa supressão do condicionamento da superfície cerâmica com ácido hidrofluorídrico ainda não parece ser uma realidade possível, devendo o clínico controlar bem o tempo de condicionamento e concentração do ácido, de forma a evitar problemas na microestrutura da restauração cerâmica.

\section{REFERÊNCIAS}

[1] R. R. Braga, R. Y. Ballester, M. R. Carrilho, Pilot study on the early shear strength of porcelain-dentin using dualcure cements, J. Prosthet. Dent. 81, 3 (1999) 285.

[2] A. M. Carneiro Júnior, R. C. R. Carvalho, M. L. Turbino, Avaliação in vitro da força de união, através de testes de tração, de porcelana feldspática com diversos tratamentos superficiais à resina composta, Rev. Odont. Univ. S. Paulo 13, 3 (1999) 257.

[3] K. Kamada, K. Yoshida, M. Atsuta, Early bond strength and durability of bond between a ceramic material and chemically-cured or dual-cured resin luting agent, Am. J. Dent. 14, 2 (2001) 85.

[4] A. Della Bona, K. J. Anusavice, Microestructure, composition and etching topography of dental ceramics, Int.

J. Prosthodont. 15, 2 (2002) 159.

[5] M. B. Blatz, A. Sadan, A. G. H. Arch Jr., B. R. Lang, In vitro evaluation of long-term bonding of Procera AllCeram alumina restorations with a modified resin luting agent, J. Prosthet. Dent. 89, 4 (2003) 381.

[6]A. Della Bona, R. Van Noort, Ceramic surface preparation for resin bonding, Am. J. Dent. 11, 6 (1998) 276.
[7] J. F. M. Pacheco, M. F. Goes, S. Consani, Influência do condicionamento e da aplicação de Silano na resistência da união porcelana- resina composta, Rev. ABO Nac. 7, 2 (1999) 81.

[8] G. A. Borges, A. M. Sophr, M. F. de Goes, L. C. Sobrinho, D. C. Chan, Effect of etching and airborne particle abrasion on the microstructure of different dental ceramics, J. Prosthet. Dent. 89, 5 (2003) 479.

[9] M. Özcan, A. Allahbeickaraghi, M. Dündar, Possible hazardous effects of hydrofluoric acid and recommendations for treatment approach: a review, Clin. Oral. Invest. 16 (2012) 15.

[10] M. T. Yucel, F. Aykent, S. Akman, I. Yondem, Effect of surface treatment methods on the shear bond strength between resin cement and all-ceramic core materials, J. Non-Crystalline Solids 358 (2012) 925.

[11] X. P. Luo, N. Silikas, M. Allaf, N. H. F. Wilson, D. C. Watts, AFM and SEM study of the effects of etching on IPS Empress 2tm dental ceramic, Surf. Sci. 491, 3 (2001) 388.

[12] T. Hooshmand, R. Van Noort, A. Keshvad, Storage effect of a pre-activated silane on the resin to ceramic bond, Dent. Mater. 20 (2004) 635.

[13] J. P. Matinlinna, L. V. Lassila, M. Ozcan, A. Yli-Urpo, P. K.Vallittu, An introduction to silanes ant their clinical applications in dentistry, Int. J. Prosthodont. 17, 32 (2004) 155.

[14] J. F. Roulet, K. J. M. Söderholm, J. Longmate, Effects of treatmentandstorage conditions on ceramic/composite bond strength, J. Dent. Res. 74, 1 (1995) 381.

[15] R. L. Myerson, Effects of silane bonding of acrylic resins to porcelain structure, J. Am. Dent. Assoc. 78, 1 (1969) 113.

[16] R. Newburg, C. H. Pameijer, Composite resins bonded to porcelain with silane solution, J. Am. Dent. Assoc. 96, 2 (1978) 288.

[17] J. R. Calamia, R. J. Simonsen, Effect of coupling agents on bond strength of etched porcelain, J. Dent. Res. 63 (1984) 62.

[18] R. Lu, J. K. Harcourt, M. J. Tyas, B. Alexander, An investigation of the composite resin/porcelain interface, Aust. Dent. J. 37, 1 (1992) 12.

[19] A. N. Özden, F. Akaltan, G. Can, Effect of surface treatments of porcelain on the shear bond strength of applied dual-cured cement, J. Prosthet. Dent. 72, 1 (1994) 85.

[20] T. Brosh, R. Pilo, N. Bichacho, R. Blutstein, Effect of combinations of surface treatments and bonding agents on the bond strength of reapired composites. J. Prosthet. Dent. 77, 2 (1997) 122.

[21] V. Ardel, M. Degrange, B. Picard, G. Derrien, Correlation of topography to bond strength of etched ceramic, Int. J. Prosthodont.12, 1 (1999) 59.

[22] S. Canay, N. Hersek, A. Ertan, Effect of different acid treatments on a porcelain surface, J. Oral Rehabil. 28 (2001) 1.

[23] W. Holland, M. Schweiger, M. Frank, V. Rheinberger, $A$ comparison of the microstructure and properties of the IPS Empress 2 and the IPS Empress glass-ceramic, J. Biomed. 
Mater. Res. 53, 4 (2000) 297.

[24] A. Della Bona, K. J. Anusavice, J. J. Mecholsky Jr., Failure analysis of resin composite bonded to ceramic, Dent. Mater.19, 8 (2003) 693.

[25] J. C. Bertoline, Hidrofuoric acid a review of toxicity, J. Emerg Med. 10 (1992) 163.

[26] G. J. Wilkes, Intravenous regional calcium gluconate for hydrofluoric acid burns of the digits, Emerg. Med. 5 (1993)155.

[27] F. Monticelli, M. Toledano, R. Osorio, M. Ferrari, Effect of temperature on the silane coupling agents when bonding core resin to quartz fiber posts, Dent. Mater. 22 (2006) 1024. [28] K. Takeuchi, M. Mizoguchi, M. Kira, M. Shimana, S. Furukawa, M. Tamura, Orientation of films prepared by evaporation of poly (dimetylsilane), J. Phys.: Condens. Matter 6 (1994) 10705.

[29] C. Shen, W. S. Oh, J. R. Williams, Effect o post- silanization drying on the bond strength of composite to ceramic, J. Prosthet. Dent. 91, 5 (2004) 453.

[30] C. N. B. Pereira, V. T. L. Buono, J. M. L. M. Mota, The influence of siane evaporation procedures on microtensile bond strength between a dental ceramic and a resin cement, Indian J. Dent. Res. 21, 2 (2010) 238.

[31] A. Meyer Filho, C. N. Souza, Desmistificando a cimentação adesiva de restaurações cerâmicas, Clínica: Int. J. Braz. Dent. 1, 1 (2005) 50.

[32] T. Nagai, Y. Kawamoto, Y. Kakehashi, H. Matsumura, Adhesive bonding of a lithium disilicate ceramic material with resin-based luting agents, J. Oral. Rehabil. 32, 8 (2005) 598.

[33] F. P. Camargo, Efeito da ciclagem térmica sobre a adesão entre uma cerâmica de dissilicato de lítio e um cimento resinoso, Pesq. Odontol. Bras. 16 (2002) 83.

(Rec. 08/02/2012, Ac. 24/04/2013) 